\title{
Review Article \\ Chronic Kidney Disease, Obesity, and Hypertension: The Role of Leptin and Adiponectin
}

\author{
M. Tesauro, ${ }^{1}$ A. Mascali, ${ }^{2}$ O. Franzese, ${ }^{3}$ S. Cipriani, ${ }^{2}$ C. Cardillo, ${ }^{4}$ and N. Di Daniele \\ ${ }^{1}$ Division of Internal Medicine, Department of Medicine of the Systems, University of Rome "Tor Vergata", Rome, Italy \\ ${ }^{2}$ Division of Nephrology, Department of Medicine of the Systems, University of Rome "Tor Vergata", Rome, Italy \\ ${ }^{3}$ Division of Pharmacology, Department of Medicine of the Systems, University of Rome "Tor Vergata", Rome, Italy \\ ${ }^{4}$ Department of Internal Medicine, "Università Cattolica del Sacro Cuore", Rome, Italy \\ Correspondence should be addressed to A. Mascali, alb.mascali@tiscali.it
}

Received 14 September 2012; Accepted 5 December 2012

Academic Editor: B. Waeber

Copyright ( 92012 M. Tesauro et al. This is an open access article distributed under the Creative Commons Attribution License, which permits unrestricted use, distribution, and reproduction in any medium, provided the original work is properly cited.

Chronic kidney disease is a major public health problem and characterized by a progressive loss in renal function over a period of months or years as defined by structural or functional abnormalities of the kidney. Several elements contribute to determine a progression of the kidney injury, inducing a worsening of renal damage and accelerating the decline of renal function: obesity and hypertension are two known factors of kidney progression. Remarkable improvements have been recently achieved in the study of the endocrine features of the adipose tissue and have been able to produce hormone-like peptides named adipokines or adipocytokines. Among these adipocytokines, which represent a link between obesity, hypertension, and chronic nephropathy, leptins and adiponectin appear to play an important role. Leptin not only is a prohypertension element (renal progression factor) through the activation sympathetic nervous, but also is able to induce prosclerotic effects directly on the kidney. In contrast, a decline of adiponectin levels has been shown to be related to a picture of hypertension: an endothelial dysfunction has been described as the main pathogenic mechanism responsible for this phenomenon.

\section{Introduction}

Chronic kidney disease is a major public health problem and characterized by a progressive loss in renal function over a period of months or years as defined by structural or functional abnormalities of the kidney. Identification and staging of chronic kidney disease are evaluated by glomerular filtration rate (GFR) and proteinuria [1].

The most important international records indicate a dramatic increase in the number of patients dependent on chronic dialysis therapy (stage 5 of chronic kidney disease) [2].

Several elements contribute to the onset and progression of chronic renal damage, including susceptibility, initiation, and progression factors.

Susceptibility elements are those situations conferring an increased risk of renal damage (age, familiarity, reduced nephron mass, low birth weight, disadvantaged social condition). Initiation elements are those factors able to determine kidney injury, especially in high-risk patients: diabetes mellitus, hypertension, autoimmune diseases, systemic infections, recurrent urinary infections, calculi/urinary tract obstruction, or drugs.

Progression elements contribute to determine a progression of the kidney injury, inducing a worsening of renal damage and accelerating the decline of renal function: (protein loss, hypertension, inadequate glycemic control of diabetic patients, cigarette smoke).

From a histological point of view, progression of renal damage is characterized mainly by glomerulosclerosis, interstitial leukocyte infiltration, and tubulointerstitial fibrosis $[3,4]$.

The link between obesity and chronic nephropathy has been initially set in the so-called theory of hyperfiltration. Nevertheless, remarkable improvements have been recently achieved in the study of the endocrine features of the adipose tissue, able to produce hormone-like peptides, named adipokines or adipocytokines. Among these adipocytokines, 
which represent a link between obesity, hypertension, and chronic nephropathy, leptins and adiponectin appear to play an important role: leptin not only is a prohypertension element (renal progression factor) through the activation sympathetic nervous, but also is able to induce prosclerotic effects directly on the kidney.

In contrast, a decline of adiponectin levels has been shown to be related to a picture of hypertension: an endothelial dysfunction has been described as the main pathogenic mechanism responsible for this phenomenon.

\section{From Hyperfiltration to the Endocrine Role of Adipose Tissue}

The theory of hyperfiltration was introduced in the last century in order to elucidate the evolution of chronic kidney disease towards the end-stage renal disease, regardless of persistence or resolution of the cause. This theory is based on the hypothesis that a critical reduction of the number of functioning nephrons would lead to altered functional and structural adaptations. Initially renal functions may be maintained, but in the long term a progressive glomerulosclerosis, proteinuria, and renal failure can be observed $[5,6]$. As a result, hemodynamic adaptations in the surviving glomeruli can be described, including vasodilation of afferent glomerular arterioles, increased filtration fraction, and increased glomerular transcapillary hydraulic pressure.

Still, these theoretical principles do not get easy application to clinical practice: a significant number of patients showing severe renal mass reduction (i.e., patients with bilateral renal carcinomas submitted to $>80 \%$ renal parenchyma resection) did not develop proteinuria or renal insufficiency in the course of followup [7].

Hyperfiltration theory has been suggested to be the cause of pandemic obesity and type 2 diabetes. As frequently demonstrated, most obese and diabetic individuals tend to show the same pattern of glomerular hemodynamics as compared to patients and animals with reduced renal mass (preglomerular vasodilation, increased glomerular filtration rate, and filtration fraction). Glomerular filtration rate (GFR) has been shown to be higher in obese individuals, while proteinuria and secondary glomerulosclerosis are now recognized as specific complications of severe obesity [3].

Huge progress has been recently achieved in the study of the endocrine features of adipose tissue, which is able to produce several hormone-like peptides named grouped adipokines. Adipose tissue contains mainly adipocytes, but also preadipocytes (not yet loaded with lipids), endothelial cells, fibroblasts, and leukocytes.

Therefore, besides its function as an energy store, a new important role is emerging for adipose tissue in the control of many pathological processes.

Several products from adipose tissue have been isolated, including adipocytokines.

The term adipocytokine is used to describe cytokines mainly produced by adipose tissue, although adipocytokines are not exclusively derived from this compartment. An important link has been shown between adiponectin, leptin, resistin and visfatin and obesity, insulin resistance, and related inflammatory disorders [8].

Most abundant adipocytokines secreted by adipocytes include adiponectin and leptin, together with nonadipocytokine immunological mediators, such as tumour- necrosis factor (TNF), interleukin-6 (IL-6), IL-1, and CC-chemokine ligand 2 (CCL2, also known as MCP1); mediators of the clotting process, such as plasminogen- activator inhibitor type 1 ; and certain complement factors providing an important link between the immune and metabolic systems.

\section{Evidences and Effects of Increased Sympathetic Activity in the Obese Patient}

Fat present around abdominal viscera in mesentery and omentum, known as visceral fat, is different from that present in subcutaneous areas (subcutaneous fat). Visceral fat induces Sympathetic Neural Activation, while subcutaneous obesity is not associated with this phenomenon. However, among obese individuals, sympathetic activation occurs almost only in those with high arterial pressure $[9,10]$. Noteworthy, Indian Pima population, characterized by obesity and hyperinsulinemia, shows low prevalence of hypertension and, in parallel, low signs of sympathetic neural activation [11].

Several evidences show an amplified sympathetic neural activation in obese patients.

Obese patients show higher serum catecholamine levels as compared to normal individuals, together with elevated noradrenaline output $[12,13]$.

Analysis of muscle sympathetic nerve activity, through the study of peroneal nerve in obese patients showing high visceral fat level, demonstrated higher "firing" frequence as compared to normal individuals [14]. Moreover, Grassi et al. [15] evidenced a decreased sympathetic activity (through muscle sympathetic nerve activity record) in obese individuals after 16 weeks of hypocaloric diet.

An increased activity of SNS could be related to upregulated renal sodium reabsorption. It can be suggested that obese children are deficient in their natriuretic response to an increased blood pressure [16]. In normal blood pressure individuals, a transitory increase in the systemic blood pressure induces higher levels of sodium and water excretion, mainly as a consequence of a reduced reabsorbance at the ascending limbo of Henle in the outer medulla. This phenomenon, known as pressure-related natriuresis, leads to a decreased effective circulating volume [17, 18], Figure 1.

These observations set the basis for the identification of a procedure of percutaneous renal sympathetic denervation $(\mathrm{RDN})$ in individuals showing metabolic syndrome and polytherapy-resistant hypertension. Although this procedure has provided encouraging results, its long-term effects must still be evaluated.

\section{Leptin}

Leptin is a adipocytokine belonging to class 1 cytokine superfamily and is abundantly produced by the adipose 


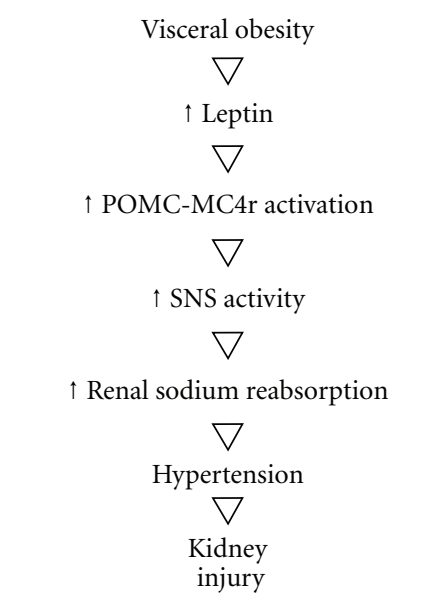

FIgURE 1: Leptin and SNS activity.

tissue. Leptin, mainly produced by visceral, subcutaneous, and pericardial adipose compartments, is also released by heart, placenta, and stomach.

Leptin receptor, a transmembrane single-chain protein, exists in 6 isoforms with a common extracellular domain and a variable length cytoplasmic portion, codified as Ob-Ra to Ob-Rf [19], where Ob-Re represents the soluble form of the receptor.

Most biological effects of leptin are mediated by the $\mathrm{Ob}-\mathrm{Rb}$ receptor, which is highly expressed at hypothalamic level. The effects of leptin on hypothalamus include the stimulation of neuropeptides regulating the balance between appetite and energetic intake, inducing satiety and increased energy consumption.

Leptin is able to activate POMC (proopiomelanocortin) expressing neurons in the curved hypothalamic nucleus, stimulating both production and release of alfa-MSH, which binds to $\mathrm{MC} 3 / \mathrm{MC} 4-\mathrm{R}$ receptors expressed on hypothalamic nuclei, inducing a reduction of appetite and an increase of energy consumption [20-22].

Da Silva et al. showed that a prolonged MC3/MC4 receptor antagonism is able to contrast leptin stimulation of SNV [23], leading to a decrease in blood pressure values.

Leptin receptors have also been isolated on endothelial [24] and smooth muscle cells (SMC) [25]. These observations suggest a role for this adipocytokine in controlling proliferation and migration of SMC and endothelial cells, thus influencing blood vessel tone and thickness of the wall. Besides, leptin has been shown to induce oxidative stress in endothelial cells [26].

It has been described how leptin levels decline during abstinence from food and increase following a number of days of hyperalimentation, when it plays its role in regulating the energetic balance.

A "resistance" mechanism has been suggested by the observation that obese patients do not show appetite reduction and increase of energy consumption in the presence of high levels of leptin [27]. This resistence to leptin does not involve all receptors, suggesting a mechanism of selective resistence, limited to the metabolic compartment and not

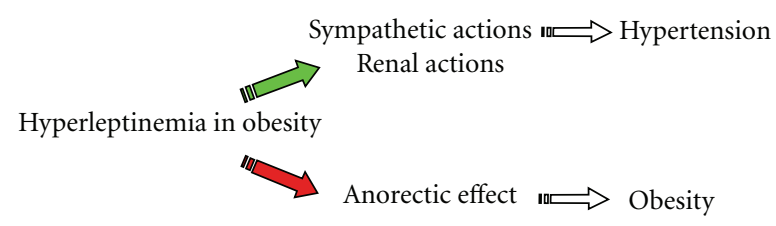

FIGURE 2: Leptin resistence.

involving other targets of the molecule, in particular ANS and kidney [27].

A plausible explanation for these observations could be a decline in the passage of leptin through the hematoencephalic barrier, resulting in the lack of the anorectic effect [28] Figure 2.

4.1. Leptin and Activation of Sympathetic Nervous System. In different experimental models, chronic hyperleptinemia has been shown to increase blood pressure. This observation could be the consequence of the activation of sympathetic nervous system as well as the impairment of natriuresis and nitric oxide inhibition [29].

Shek et al. demonstrated that a chronic infusion of leptin in mice was able to induce a persistent rise in blood pressure, which returned to normal values following the suspension of the infusion [30].

Aizawa-Abe et al. [31] showed an increase of about $20 \mathrm{mmHg}$ in systolic pressure in leptin hyperexpressing transgenic mice showing elevated urinary catecholamine excretion.

Moreover, da Silva et al. evidenced that obesity is associated with an increased sympathetic activity, in particular in the renal compartment, while the critical role of renal innervation was demonstrated in dogs through bilateral denervation techniques [32].

Prior et al. were able to identify a close relationship between plasmatic leptin concentration and renal sympathetic activity [33]. The critical role for leptin in obesitylinked hypertension has also been demonstrated by Rahmouni et al. In this study, conducted in 3 murine models resembling human Bardet-Biedle syndrome, leptin was able to induce upregulation of renal sympathetic activity and increased blood pressure values in 2 models out of 3 , while blood pressure returned to normal values following gangliar block [34].

4.2. Leptin and Kidney Damage. The effects leptin is potentially able to exert on the kidney may contribute to the worsening of the renal function. Studies conducted in murine models have shown how hyperexpression of lectin increases the activity of sympathetic nervous system, leading to an upregulation of blood pressure and an increase of urinary catecholamine excretion $[31,35]$. A small amount of the isoform $\mathrm{Ob}-\mathrm{RB}$ receptor is expressed on the kidney, which on the contrary expresses a large amount of the shorter Ob-Ra isoform [36]. Leptin has been shown to induce the growth of cultured glomerular endothelial cells and increase the production of transforming-growth-factor-(TGF-) beta 1 . 
No much information is available about the influence of leptin on the podocyte. A $72 \mathrm{~h}$ infusion of recombinant leptin is able to induce TGF- alfa 1 expression and increases the total number of proliferating cells, while a three-week infusion is associated with an amplified glomerular expression of type IV collagen [37]. Raised levels of blood pressure were not evidenced, while a significant increase in urinary protein excretion was experienced.

Leptin induces the synthesis of type 1 collagen in mesangial cells, as well as type 4 collagen in glomerular endothelial cells contributing to extracellular matrix deposition, glomerulosclerosis, and proteinuria [38].

A link has been described between the increased incidence of glomerulosclerosis in patients with severe obesity and serum leptin concentration.

Usually, obese patients show increased risk of developing glomerulosclerosis. In particular, proteinuria and chronic kidney disease can be observed following unilateral nephrectomy [39].

$92 \%$ of the obese patients with body mass indexes (BMIs) greater than 30 developed proteinuria and renal insufficiency as compared with only $12 \%$ of those patients with BMIs less than 30 [40].

It has been suggested that an increase of extracellular matrix production can also be mediated by a cooperation of leptin with other soluble mediators in order to promote diabetic nephropathy. High serum levels of leptin have been described in obese hyperinsulinemic type 2 diabetic patients.

In Pima Indians, a correlation has been described between increased urine leptin levels and augmented albuminuria, while a negative correlation was established between high urine leptin levels and GFR [41]. Patients affected by type 2 diabetes showed a link between higher serum leptin concentrations and increased urine albumin excretion. Moreover, in a model of type 2 diabetic mice, the $\mathrm{db} / \mathrm{db}$, the development of glomerular hypertrophy, glomerulosclerosis, renal insufficiency, and eventually proteinuria have been described. Noteworthy, it has been shown how neutralization of TGF-beta by specific monoclonal antibody is able to prevent the expansion of mesangial renal and renal insufficiency. Moreover, while a mesangial expansion has been observed in the hyperleptinemic $\mathrm{db} / \mathrm{db}$ mice,renal disease is only rarely observed in ob/obmice, who are simply leptin deficient.

\section{Adiponectin}

Adiponectin is a $30 \mathrm{kDa}$ protein mainly secreted by adipocytes. It has been described how obesity is associated to hypoadiponectinemia, probably through inhibition of gene transcription and a reduced release of the factor [42].

5.1. Adiponectin and Hypertension. Several clinical studies have established a relationship between adiponectin plasma concentration and hypertension.

Adiponectin plasma levels have been shown to be significantly lower in patients affected by essential hypertension as compared with individuals with normal blood pressure [43].
An inverse correlation has been observed between meandiastolic pressure and adiponectin concentration.

Hypoadiponectinemia is a risk factor for arterial hypertension, independently from a potential condition of insulin resistance and diabetes [44].

Chow et al. [45] showed a correlation between a decline of adiponectin concentration and the risk of developing hypertension in normal individuals over a period of 5 years.

Studies on adiponectin genetic variants have provided several information about the link between hypertension and adiponectin.

The polymorphism 164 in the gene encoding for adiponectin has been associated with hypoadiponectinemia and arterial hypertension in Japanese individuals [44].

Experimental studies support this role for adiponectin in regulating arterial blood pressure [46]: adiponectin knockout mice do not show hypertension signs in the absence of stress inducing factors, while showing higher expression values as compared with wild type mice following a lowsodium content diet.

In uninephrectomy lineages subjected to infusion with aldosterone, adiponectin knockout subtypes developed higher arterial pressure values as compared with wild types [47] while showing a more severe diastolic cardiac dysfunction.

5.2. Adiponectin and Endothelial Function. Endotelial dysfunction is a major predisposing factor for vasculopathy and is strictly associated with compliances related to obesity such as insulin resistance and arterial hypertension [48].

Normal levels of adiponectin seem to be fundamental for a physiological endothelial function; adiponectin plasma levels are strictly related to the vasodilatatory response. Hypoadiponectinemia has been associated with a decline of brachial arterial vasodilatatory response in diabetic patients [49]. Similarly, adiponectin knockout mice show a reduced vasodilatatory response to acetylcholine as compared with controls [50].

The endothelial enzyme nitric oxide synthase (eNOS) and NO control the vascular homeostasis and, in particular, the endothelial function $[51,52]$.

Adiponectin has been suggested to modulate the endogenous production of NO by endothelial cells.

Adiponectin knockout mice show lower amount of eNOS at the level of aortic endothelium and lower plasma NO metabolite levels [46], together with higher arterial pressure levels as compared with controls.

Nishimura et al. [53] evidenced a more severe ischemiareperfusion-related damage in knock out mice, together with a decline of eNOS levels in the encephalon.

In vitro studies have confirmed the role of adiponectin in regulating eNOS activity and NO production. Adiponectin stimulates phosphorylation of eNOS at serine 1177 in human endothelial cells, through the activation of kinases [54], therefore inducing an NO production [55].

Activation of the kinase cascade follows a possible interaction between adiponectin and AdipoR1- and Adipo $\mathrm{R} 2$-specific receptors [56]. 
Adiponectin also plays a role in stimulating the endothelial cyclooxygenase 2 (cox-2) with production of $\mathrm{PGI}_{2}$, showing vasodilatatory action. Interaction between adiponectin and endothelial cells has been shown to be mediated by the complex calreticulin/CD91.

Therefore, adiponectin plays an important role in regulating endothelial function through the stimulation of eNOS as well as $\mathrm{PGI}_{2}$.

5.3. Adiponectin and Kidney. Several clinical studies have also demonstrated a link between hypoadiponectinemia and microalbuminuria in patients showing hypertension, as well as Japanese [57] and Afro-American obese patients [58].

Sharma et al. evidenced [59] the highest basal levels of microalbuminuria in adiponectin knockout mice, together with an alteration of podocyte pediculi.

A recent study by Kacso et al. [60], conducted on 86 type 2 diabetic patients, with a GFR > $30 \mathrm{ML} / \mathrm{MIN}$, has demonstrated a more evident progression of renal damage in patients characterized by hypoadiponectinemia as compared with controls.

\section{Conclusions}

Characterization of the endocrine features of fat tissue has radically modified the physiopathological view of related syndromes such as obesity, arterial hypertension and chronic renal disease. Among adipocytokines specifically produced by fat tissue, leptin and adipokine play a central role in the genesis of hypertension and renal damage. Leptins represent a crucial cause of hypertension and are important initiation and progression factors for chronic renal failure, through abnormal sympathetic neural stimulation, showing a direct prothrombotic effect on the kidney. Adiponectinemia is associated with hypertension and endothelial disfunction.

Further studies are necessary in order to elucidate the mechanisms involved in adipocytokines-mediated role in chronic kidney disease.

\section{References}

[1] "K/DOQI clinical practice guideline for chronic kidney disease: evaluation, classification and stratification," American Journal of Kidney Diseases, vol. 39, supplement 2, article 246, 2002.

[2] J. Coresh, E. Selvin, L. A. Stevens et al., "Prevalence of chronic kidney disease in the U.S. during 1988-1994 and 1999-2004," The Journal of the American Medical Association, vol. 298, no. 17, pp. 2038-2047, 2007.

[3] D. O. Schlondorff, "Overview of factors contributing to the pathophysiology of progressive renal disease," Kidney International, vol. 74, pp. 860-866, 2008.

[4] M. Abbate, C. Zoja, and G. Remuzzi, "How does proteinuria cause progressive renal damage?" Journal of the American Society of Nephrology, vol. 17, no. 11, pp. 2974-2984, 2006.

[5] T. H. Hostetter, J. L. Olson, H. G. Rennke et al., "Hyperfiltration in remnant nephrons: a potentially adverse response to renal ablation," American Journal of Physiology, vol. 10, no. 1, pp. F85-F93, 1981.
[6] B. M. Brenner, T. W. Meyer, and T. H. Hostetter, "Dietary protein intake and the progressive nature of kidney disease: the role of hemodynamically mediated glomerular injury in the pathogenesis of progressive glomerular sclerosis in aging, renal ablation, and intrinsic renal disease," New England Journal of Medicine, vol. 307, no. 11, pp. 652-659, 1982.

[7] E. González, E. Gutiérrez, E. Morales et al., "Factors influencing the progression of renal damage in patients with unilateral renal agenesis and remnant kidney," Kidney International, vol. 68, no. 1, pp. 263-270, 2005.

[8] H. Tilg and A. R. Moschen, "Adipocytokines: mediators linking adipose tissue, inflammation and immunity," Nature Reviews Immunology, vol. 6, no. 10, pp. 772-783, 2006.

[9] G. E. Alvarez, T. P. Ballard, S. D. Beske, and K. P. Davy, "Subcutaneous obesity is not associated with sympathetic neural activation," American Journal of Physiology, vol. 287, no. 156-1, pp. H414-H418, 2004.

[10] G. E. Alvarez, S. D. Beske, T. P. Ballard, and K. P. Davy, "Symphatetic neural activation in visceral obesity," Circulation, vol. 106, no. 20, pp. 2533-2536, 2002.

[11] C. Weyer, R. E. Pratley, S. Snitker, M. Spraul, E. Ravussin, and P. A. Tataranni, "Ethnic differences in insulinemia and sympathetic tone as links between obesity and blood pressure," Hypertension, vol. 36, no. 4, pp. 531-537, 2000.

[12] I. C. Trombetta, L. T. Batalha, M. U. P. B. Rondon et al., "Weight loss improves neurovascular and muscle metaboreflex control in obesity," American Journal of Physiology, vol. 285, no. 3, pp. H974-H982, 2003.

[13] M. Vaz, G. Jennings, A. Turner, H. Cox, G. Lambert, and M. Esler, "Regional sympathetic nervous activity and oxygen consumption in obese normotensive human subjects," Circulation, vol. 96, no. 10, pp. 3423-3429, 1997.

[14] G. Grassi, R. Dell'Oro, A. Facchini, F. Q. Trevano, G. B. Bolla, and G. Mancia, "Effect of central and peripheral body fat distribution on sympathetic and baroreflex function in obese normotensives," Journal of Hypertension, vol. 22, no. 12, pp. 2363-2369, 2004.

[15] G. Grassi, G. Seravalle, M. Colombo et al., "Body weight reduction, sympathetic nerve traffic, and arterial baroreflex in obese normotensive humans," Circulation, vol. 97, no. 20, pp. 2037-2042, 1998.

[16] E. Lurbe, V. Alvarez, Y. Liao et al., "Obesity modifies the relationship between ambulatory blood pressure and natriuresis in children," Blood Pressure Monitoring, vol. 5, no. 5-6, pp. 275-280, 2000.

[17] A. W. Cowley, "Renal medullary oxidative stress, pressurenatriuresis, and hypertension," Hypertension, vol. 52, no. 5, pp. 777-786, 2008.

[18] E. E. Selkurt, "Effect of pulse pressure and mean arterial pressure modification on renal hemodynamics and electrolyte and water excretion," Circulation, vol. 4, no. 4, pp. 541-551, 1951.

[19] L. A. Tartaglia, “The leptin receptor," The Journal of Biological Chemistry, vol. 272, no. 10, pp. 6093-6096, 1997.

[20] M. G. Castro and E. Morrison, "Post-translational processing of pro-opiomelanocortin in the pituitary and in the brain," Critical Reviews in Neurobiology, vol. 11, no. 1, pp. 35-57, 1997.

[21] M. W. Schwartz, S. C. Woods, D. Porte Jr., R. J. Seeley, and D. G. Baskin, "Central nervous system control of foof intake," Nature, vol. 404, no. 6778, pp. 661-671, 2000.

[22] B. M. Spiegelman and J. S. Flier, "Obesity and the regulation of energy balance," Cell, vol. 104, no. 4, pp. 531-543, 2001. 
[23] A. A. da Silva, J. J. Kuo, and J. E. Hall, "Role of hypothalamic melanocortin 3/4-receptors in mediating chronic cardiovascular, renal, and metabolic actions of leptin," Hypertension, vol. 43, no. 6, pp. 1312-1317, 2004.

[24] M. R. Sierra-Honigmann, A. K. Nath, C. Murakami et al., "Biological action of leptin as an angiogenic factor," Science, vol. 281, no. 5383, pp. 1683-1686, 1998.

[25] A. Oda, T. Taniguchi, and M. Yokoyama, "Leptin stimulates rat aortic smooth muscle celle proliferation and migration," Kobe Journal of Medical Sciences, vol. 47, no. 3, pp. 141-159, 2001.

[26] S. I. Yamagishi, D. Edelstein, X. L. Du, Y. Kaneda, M. Guzmán, and M. Brownlee, "Leptin induces mitochondrial superoxide production and monocyte chemoattractant protein-1 expression in aortic endothelial cells by increasing fatty acid oxidation via protein kinase A," The Journal of Biological Chemistry, vol. 276, no. 27, pp. 25096-25100, 2001.

[27] J. P. Montani, V. Antic, Z. Yang, and A. Dulloo, "Pathways from obesity to hypertension: from the perspective of a vicious triangle," International Journal of Obesity, vol. 26, supplement 2, pp. S28-S38, 2002.

[28] D. L. Morris and L. Rui, "Recent advances in understanding leptin signaling and leptin resistance," American Journal of Physiology, vol. 297, no. 6, pp. E1247-E1259, 2009.

[29] N. Werner and G. Nickenig, "From fat fighter to risk factor: rhe zigzag trek of leptin," Arteriosclerosis, Thrombosis, and Vascular Biology, vol. 24, no. 1, pp. 7-9, 2004.

[30] E. W. Shek, M. W. Brands, and J. E. Hall, "Chronic leptin infusion increases artrial pressure," Hypertension, vol. 31, pp. 409-414, 1998.

[31] M. Aizawa-Abe, Y. Ogawa, H. Masuzaki et al., "Pathophysiological role of leptin in obesity-related hypertension," Journal of Clinical Investigation, vol. 105, no. 9, pp. 1243-1252, 2000.

[32] A. A. da Silva, J. do Carmo, J. Dubinion, and J. E. Hall, "The role of the sympathetic nervous system in obesity-related hypertension," Current Hypertension Reports, vol. 11, no. 3, pp. 206-211, 2009.

[33] L. J. Prior, N. Eikelis, J. A. Armitage et al., "Exposure to a highfat diet alters leptin sensitivity and elevates renal sympathetic nerve activity and arterial pressure in rabbits," Hypertension, vol. 55, no. 4, pp. 862-868, 2010.

[34] K. Rahmouni, M. A. Fath, S. Seo et al., "Leptin resistance contributes to obesity and hypertension in mouse models of Bardet-Biedl syndrome," Journal of Clinical Investigation, vol. 118, no. 4, pp. 1458-1467, 2008.

[35] W. G. Haynes, D. A. Morgan, S. A. Walsh, A. L. Mark, and W. I. Sivitz, "Receptor-mediated regional sympathetic nerve activation by leptin," Journal of Clinical Investigation, vol. 100, no. 2, pp. 270-278, 1997.

[36] C. Serradeil-Le Gal, D. Raufaste, G. Brossard et al., "Characterization and localization of leptin receptors in the rat kidney," FEBS Letters, vol. 404, no. 2-3, pp. 185-191, 1997.

[37] G. Wolf, A. Hamann, D. C. Han et al., "Leptin stimulates proliferation and TGF- $\beta$ expression in renal glomerular endothelial cells: potential role in glomerulosclerosis," Kidney International, vol. 56, no. 3, pp. 860-872, 1999.

[38] B. J. Ballerman, "A role for leptin in glomerulosclerosis?" Kidney International, vol. 56, no. 3, pp. 1154-1155, 1999.

[39] B. L. Kasiske and J. T. Crosson, "Renal disease in patients with massive obesity," Archives of Internal Medicine, vol. 146, no. 6, pp. 1105-1109, 1986.

[40] M. Praga, E. Hernandez, J. C. Herrero et al., "Influence of obesity on the appearance of proteinuria and renal insufficiency after unilateral nephrectomy," Kidney International, vol. 58, no. 5, pp. 2111-2118, 2000.
[41] C. Wilson, R. Nelson, M. Nicolson, and R. Pratley, "Plasma leptin concentrations: no difference between diabetic pima indians with and without nephropathy," Diabetologia, vol. 41, no. 7, pp. 861-862, 1998.

[42] S. A. Phillips, T. P. Ciaraldi, D. K. Oh, M. K. Savu, and R. R. Henry, "Adiponectin secretion and response to pioglitazone is depot dependent in cultured human adipose tissue," American Journal of Physiology, vol. 295, no. 4, pp. E842-E850, 2008.

[43] M. Adamczak, A. Więcek, T. Funahashi, J. Chudek, F. Kokot, and Y. Matsuzawa, "Decreased plasma adiponectin concentration in patients with essential hypertension," American Journal of Hypertension, vol. 16, no. 1, pp. 72-75, 2003.

[44] Y. Iwashima, T. Katsuya, K. Ishikawa et al., "Hypoadiponectinemia is an independent risk factor for hypertension," Hypertension, vol. 43, no. 6, pp. 1318-1323, 2004.

[45] W. S. Chow, B. M. Y. Cheung, A. W. K. Tso et al., "Hypoadiponectinemia as a predictor for the development of hypertension: a 5-year prospective study," Hypertension, vol. 49, no. 6, pp. 1455-1461, 2007.

[46] K. Ohashi, S. Kihara, N. Ouchi et al., "Adiponectin replenishment ameliorates obesity-related hypertension," Hypertension, vol. 47, no. 6, pp. 1108-1116, 2006.

[47] F. Sam, T. A. S. Duhaney, K. Sato et al., "Adiponectin deficiency, diastolic dysfunction, and diastolic heart failure," Endocrinology, vol. 151, no. 1, pp. 322-331, 2010.

[48] T. F. Luscher, "The endothelium and cardiovascular diseasea complex relation," The New England Journal of Medicine, vol. 330, no. 15, pp. 1081-1083, 1994.

[49] K. C. B. Tan, A. Xu, W. S. Chow et al., "Hypoadiponectinemia is associated with impaired endothelium-dependent vasodilation," Journal of Clinical Endocrinology and Metabolism, vol. 89, no. 2, pp. 765-769, 2004.

[50] N. Ouchi, M. Ohishi, S. Kihara et al., "Association of hypoadiponectinemia with impaired vasoreactivity," Hypertension, vol. 42, no. 3, pp. 231-234, 2003.

[51] P. L. Huang, Z. Huang, H. Mashimo et al., "Hypertension in mice lacking the gene for endothelial nitric oxide synthase," Nature, vol. 377, no. 6546, pp. 239-242, 1995.

[52] T. J. Guzik, E. Black, N. E. West et al., "Relationship between the G894T polymorphism (Glu 298 Asp variant) in endothelial nitric oxide synthase and nitric oxide-mediated endothelial function in human atherosclerosis," American Journal of Medical Genetics, vol. 100, no. 2, pp. 130-137, 2001.

[53] M. Nishimura, Y. Izumiya, A. Higuchi et al., "Adiponectin prevents cerebral ischemic injury through endothelial nitric oxide synthase-dependent mechanisms," Circulation, vol. 117, no. 2, pp. 216-223, 2008.

[54] N. Ouchi, H. Kobayashi, S. Kihara et al., "Adiponectin stimulates angiogenesis by promoting cross-talk between AMPactivated protein kinase and Akt signaling in endothelial cells," The Journal of Biological Chemistry, vol. 279, no. 2, pp. 13041309, 2004.

[55] H. Chen, M. Montagnani, T. Funahashi, I. Shimomura, and M. J. Quon, "Adiponectin stimulates production of nitric oxide in vascular endothelial cells," The Journal of Biological Chemistry, vol. 278, no. 45, pp. 45021-45026, 2003.

[56] K. K. Y. Cheng, K. S. L. Lam, Y. Wang et al., "Adiponectininduced endothelial nitric oxide synthase activation and nitric oxide production are mediated by APPL1 in endothelial cells," Diabetes, vol. 56, no. 5, pp. 1387-1394, 2007.

[57] C. Tsioufis, K. Dimitriadis, D. Chatzis et al., "Relation of microalbuminuria to adiponectin and augmented C-reactive protein levels in men with essential hypertension," American Journal of Cardiology, vol. 96, no. 7, pp. 946-951, 2005. 
[58] Y. Yano, S. Hoshide, J. Ishikawa et al., "Differential impacts of adiponectin on low-grade albuminuria between obese and nonobese persons without diabetes," Journal of Clinical Hypertension, vol. 9, no. 10, pp. 775-782, 2007.

[59] K. Sharma, S. RamachandraRao, G. Qiu et al., "Adiponectin regulates albuminuria and podocyte function in mice," Journal of Clinical Investigation, vol. 118, no. 5, pp. 1645-1656, 2008.

[60] I. M. Kacso, C. I. Bondor, and G. Kacso, "Plasma adiponectin is related to the progression of kidney disease in type 2 diabetes patients," Scandinavian Journal of Clinical and Laboratory Investigation, vol. 72, no. 4, pp. 333-339, 2012. 


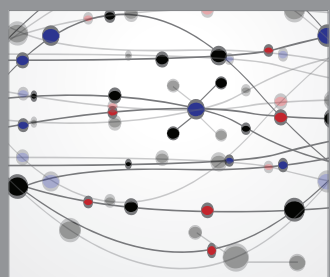

The Scientific World Journal
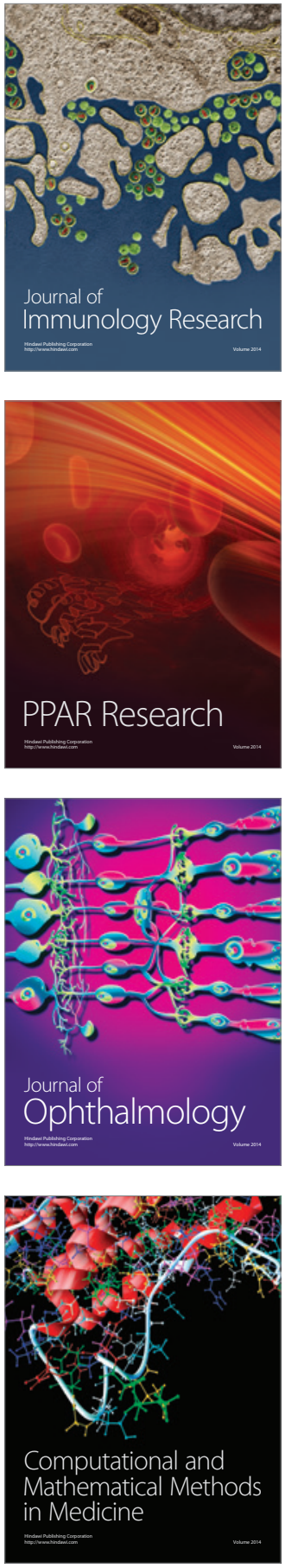

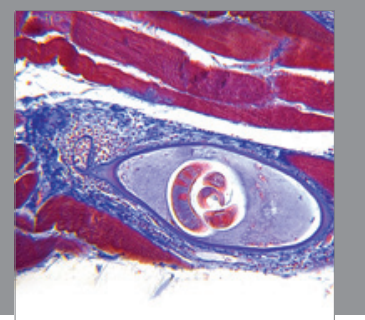

Gastroenterology

Research and Practice
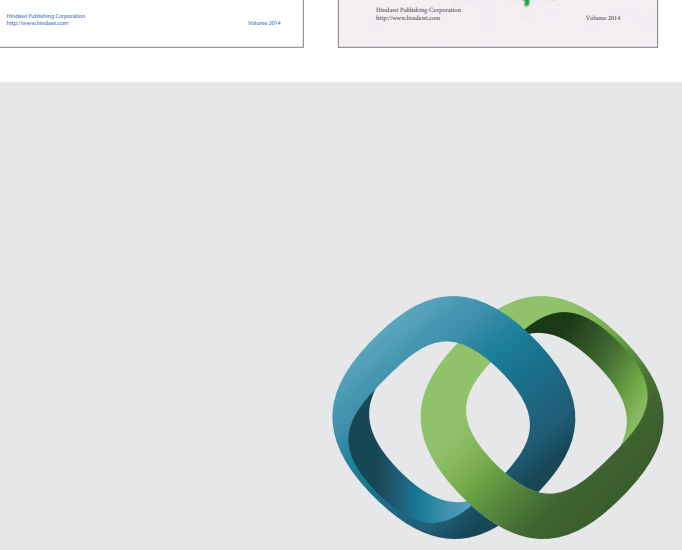

\section{Hindawi}

Submit your manuscripts at

http://www.hindawi.com
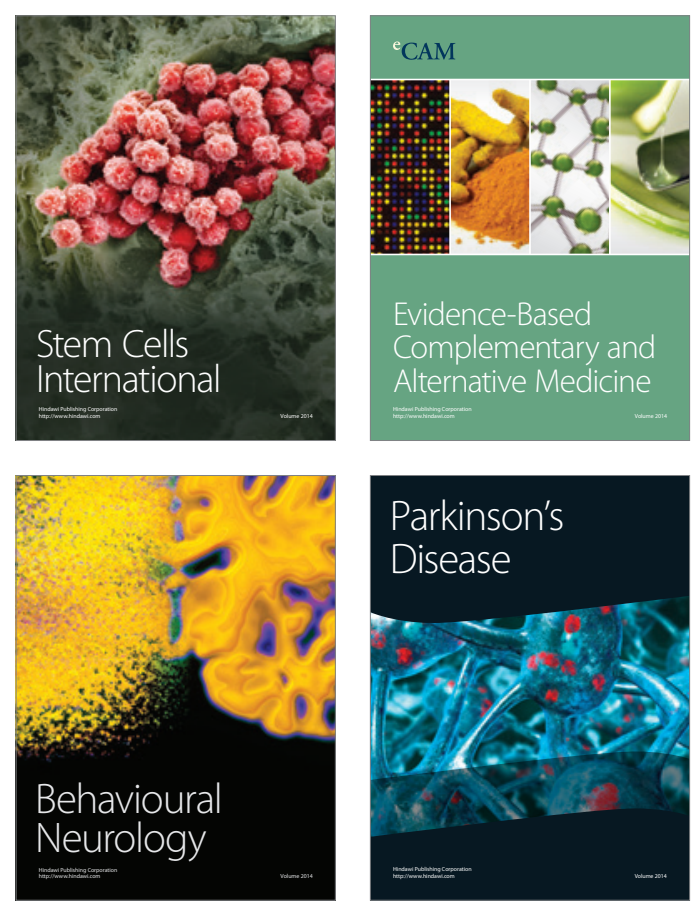

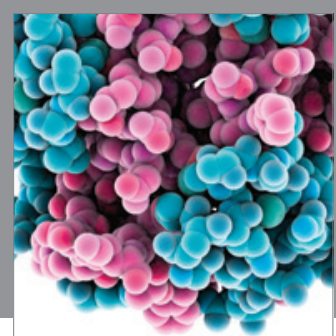

Journal of
Diabetes Research

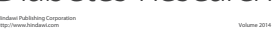

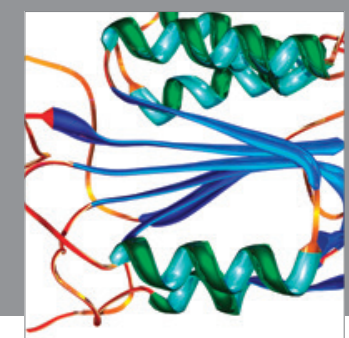

Disease Markers
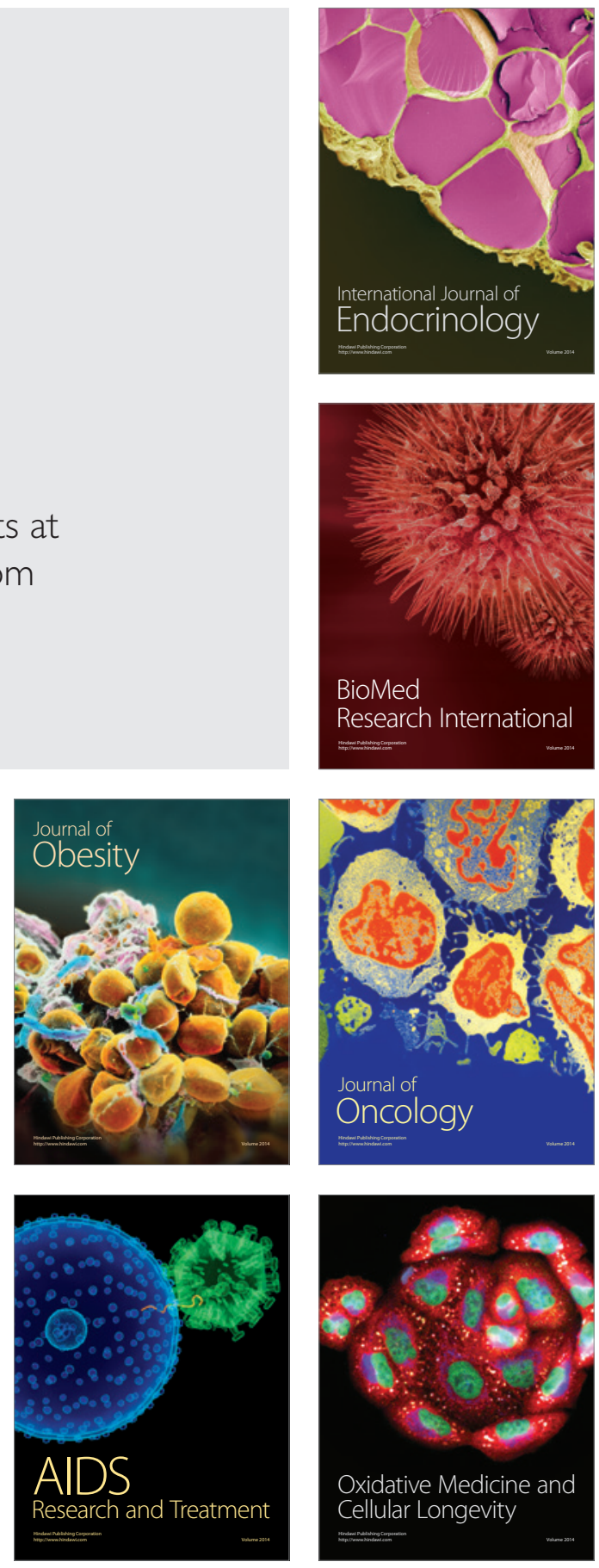